\title{
Estudo Comparativo da Autonomia de Ação de Idosas Praticantes e Não Praticantes de Exercícios Físicos Regulares
}

\author{
Comparative Study on the Autonomy of Female Elderly Practitioners \\ and Non-Practitioners of Regular Physical Exercises
}

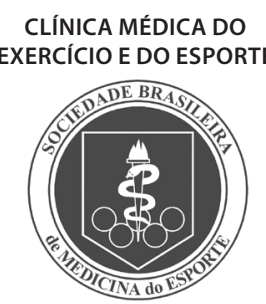

Artigo Original

\begin{abstract}
Anselmo José Perez'
Otávio Tavares'

Flor Bonadiman Fusi

Gabriela Linhares Daltio'

Paulo de Tarso Veras Farinatti2,3

1. Centro de Educação Física e Desportos - Universidade Federal do Espírito Santo, Vitória, ES, Brasil. 2. Laboratório de Atividade Física e Promoção da Saúde, Instituto de Educação Física e Desportos, Universidade do Estado do Rio de Janeiro, Rio de Janeiro, RJ, Brasil.

3. Mestrado em Ciências da

Atividade Física, Universidade Salgado de Oliveira, Niterói, RJ, Brasil.
\end{abstract}

\section{Endereço para correspondência:} Universidade Federal do Espírito Santo

Centro de Educação Física e

Desportos

Av. Fernando Ferrari, no 514,

Goiabeiras - 29075-910 - Vitória,

ES, Brasil

E-mail: anselmo@cefd.ufes.br

\begin{abstract}
RESUMO
A prática regular de exercícios físicos pode afetar favoravelmente a autonomia. O estudo comparou a autonomia de ação de idosas praticantes ( $G E, n=27)$ e não praticantes $(G N E, n=21)$ de exercícios, valendo-se do Sistema Sênior de Avaliação da Autonomia de Ação (SysSen). O SysSen é composto por questionário (QSAP), voltado para necessidades de potência aeróbia (PA) e força (FO) em atividades compatíveis com uma vida percebida como autônoma, e teste de campo (TSMP), no qual se caminha 800m transportando cargas predeterminadas. O QSAP fornece o índice de autonomia exprimida (IAE) e o TSMP o índice de autonomia potencial (IAP) que, cruzados, definem o índice de autonomia de ação (IAP/IAE =ISAC). Um ISAC $\geq 1,0$ caracteriza o sujeito como autônomo. Os grupos eram similares quanto à idade (GNE $=68 \pm 5 \mathrm{vs}$. GE $=69 \pm 6$ anos; $p=0,56$ ), peso $(\mathrm{GNE}=65,7 \pm 14,6$ vs. GE $=67,4 \pm 10,1 \mathrm{~kg} ; \mathrm{p}=0,65)$ e estatura (GNE =1,54 $\pm 0,05 \mathrm{vs}$. GE =1,56 $\pm 0,04 \mathrm{~m} ; \mathrm{p}=$ 0,28). Houve diferenças significativas para o tempo de execução do TSMP (GNE $=678,9 \pm 78,0$ vs. GE $=631,0 \pm$ $91,1 \mathrm{~s} ; \mathrm{p}=0,05)$ e IAP ( $G N E=64,4 \pm 5,2$ vs. $G E=68,2 \pm 4,7 ; p=0,01)$. No tocante ao QSAP, não se constataram diferenças significativas para o IAE (GNE =73,3 $\pm 57,0$ vs. GE $=73,7 \pm 5,1 ; p=0,85$ ) ou necessidades de FO e PA. Todavia, os resultados para PA e FO foram maiores para GE nos itens relacionados às atividades que as idosas faziam efetivamente $(p<0,05)$. Não houve diferença entre os grupos para o ISAC $(p=0,55)$. Conclui-se que a prática regular de exercícios não influenciou significativamente a autonomia de ação apreciada pelo SysSen, provavelmente em virtude de elevação proporcional do IAE e IAP nos grupos ativo e inativo.
\end{abstract}

Palavras-chave: envelhecimento, autonomia funcional, atividade física, aptidão física, saúde.

\begin{abstract}
Regular exercise practice is favorably related with functional autonomy. The aim of this study was to investigate whether the physical activity level influences on the results of the Senior System for Assessment of Action Autonomy (SysSen) in elderly women. The SysSen is composed of a questionnaire (QSAP) focusing on the needs of aerobic power (AP) and upper limb strength (S) for a life perceived as autonomous, and a field test (TSMP), in which the subject walks $800 \mathrm{~m}$ carrying pre-set loads. The QSAP provides the Expressed Autonomy Index (EAI) and the TSMP, the Potential Autonomy Index (PAI). The ratio between EAI and PAI defines the Autonomy of Action Index (PAI/EAI = AAI). An AAlof 1.0 or more characterizes the subject as independent. The subjects were either assigned to physically active ( $E G, n=27$ ) or non-active groups ( $N E G, n=21)$, which were similar in age (NEG = $68 \pm 5$ vs $\mathrm{EG}=69 \pm 6$ years; $p=0.56$ ), weight ( $\mathrm{NEG}=65.7 \pm 14.6$ vs $\mathrm{EG}=67.4 \pm 10.1 \mathrm{~kg} ; \mathrm{p}=0.65)$, and height (NEG $=1.54 \pm 0.05 \mathrm{vs} E G=1.56 \pm 0.04 \mathrm{~m} ; \mathrm{p}=0.28$ ). No differences were found for the $\mathrm{EAI}$ (NEG $73.3 \pm 57.0 \mathrm{vs} E G=$ $73.7 \pm 5.1 ; p=0.85$ ) or needs related to AP and $S$. However, the results for AP and $S$ in $E G$ were higher in the items concerned with the activities daily performed $(p<.05)$. The AAI was statistically similar across the groups $(p=$ 0.55). In conclusion, regular exercise practice did not influence on the action autonomy as appreciated by the SysSen, probably due to a proportional increase of EAI and PAI in the active and inactive groups.
\end{abstract}

Keywords: aging, autonomy, physical activity, physical fitness, health.

\section{INTRODUÇÃO}

A prática regular de atividades físicas é tida como estratégia auxiliar para redução do impacto do envelhecimento sobre a autonomia funcional e qualidade de vida ${ }^{(1-4)}$. Por outro lado, é comum associar-se a autonomia às incapacidades desencadeadoras de dependência física e psíquica ${ }^{(5)}$, desconsiderando os problemas ambientais, sociais, culturais e econômicos que, seguramente, em maior ou menor extensão participam do processo(6) ${ }^{(6)}$ Muitos autores têm estudado sob diferentes ângulos a relação entre o exercício físico regular e os níveis de autonomia/independência do idoso, quer nos seus aspectos biológicos ${ }^{(4,5,7-12)}$, quer em aspectos psicossocioculturais $(2,3,12,13)$.

A autonomia dos idosos é, não raro, associada à mera ausência de incapacidades estabelecidas clinicamente; entende-se que, da mesma forma que a saúde não é ausência de doença, a autonomia não é ausência de dependência física ${ }^{(14)}$. Avaliar a autonomia sob um ângulo positivo é compreender os modos de vida pessoais e coletivos, através de mudanças nas atitudes de valores e da multiplicação do conhecimento e informação, permitindo que o idoso reja sua vida de acordo com suas necessidades, anseios, adaptações, realizações pessoais, enfim, sendo realmente autocapaz.

Com base nessa premissa, Farinatti ${ }^{(15)}$ apresentou uma proposta para avaliar a autonomia de idosos, que permitiria levar em conta fatores físicos, ambientais e emocionais relacionados às atividades tidas como importante pelo idoso para uma vida autônoma. Esse sistema de avaliação, denominado Sistema Sênior de Avaliação da Autonomia de Ação (SysSen), vem sendo objeto de estudos que procuram atestar sua validade ${ }^{(14-17)}$. 
Os resultados obtidos até aqui pelos estudos de validação indicam que o SysSen traduz convenientemente as relações entre demandas e potencial físico individual para uma vida autônoma, considerando aspectos positivos e negativos da autonomia de ação com bons níveis de pertinência e de reprodutibilidade. Todavia, há a necessidade de esforços adicionais para verificação da estabilidade e consistência dos resultados fornecidos pelo método em diferentes grupos populacionais. Por exemplo, ainda são escassos estudos comparativos em populações brasileiras ${ }^{(16)}$. Sendo assim, o objetivo do presente estudo foi comparar os resultados do SysSen em idosas praticantes e não praticantes de exercícios físicos regulares da cidade de Vitória, Espírito Santo, Brasil.

\section{MÉTODOS}

\section{Amostragem}

A amostra caracterizou-se como não-probabilística(18), constituindose de mulheres com mais de 60 anos de idade. Os critérios adotados para exclusão da participação no estudo foram: 1) restrição médica à prática de exercício; 2) problemas osteomioarticulares que restringissem a realização do teste; 3 ) diagnóstico de hipertensão arterial (sistólica > 140mmHg e diastólica > 90mmHg); 4) deficiência motora ou cognitiva que impossibilitasse as respostas ao questionário ou execução do teste de campo. Participaram do estudo 48 idosas distribuídas em dois grupos. Um deles realizava exercícios físicos regularmente há pelo menos seis meses (grupo exercício $-G E, n=27$, idade $=69 \pm 6$ anos), enquanto o outro estava há pelo menos seis meses sem praticar exercícios (grupo não-exercício - $\mathrm{GNE}, \mathrm{n}=21$, idade $=68 \pm 5$ anos).

No $G E$, as atividades realizadas pelas idosas eram: hidroginástica ( $n=$ 14), caminhada ( $n=15)$, alongamento $(n=13)$, ginástica tradicional $(n=$ 8), ginástica aeróbia de baixo impacto $(n=2)$, dança $(n=9)$, musculação $(n=2)$ e yoga $(n=4)$, sendo a maioria $(n=15)$ participante do Serviço de Orientação ao Exercício da Prefeitura Municipal de Vitória (SOE). Todas as participantes da pesquisa, após esclarecimento do objetivo e características do estudo, assinaram o Termo de Consentimento Pós-informado, conforme estabelecido pela Resolução 196/96 do Conselho Nacional de Saúde para Pesquisas Envolvendo Seres Humanos. O estudo foi aprovado pelo Comitê de Ética institucional (CEP-UFES, nº 101/08).

\section{PROCEDIMENTOS}

A autonomia de ação foi aferida pelo SysSen, descrito detalhadamente em publicações prévias, quanto ao seu desenvolvimento, validação e protocolo de aplicação(15,19,20). O sistema é formado por um questionário (QSAP) e um teste de campo (TSMP). Para aplicação do QSAP eram entregues ao entrevistado folhas com respostas possíveis a todas as questões, cabendo ao entrevistador fazer as questões com base em matriz mais complexa, na qual a cotação do instrumento era posteriormente calculada ${ }^{(14)}$. Através do QSAP obtinham-se índices representativos das necessidades individuais quanto à potência aeróbia (PA) e força (FO) de membros superiores, relacionadas às atividades físicas levantadas como importantes para a vida autônoma nas seguintes dimensões: Parte I - O que o entrevistado faz; Parte II - O que o entrevistado deve fazer; Parte III - O que o entrevistado deseja fazer; Parte IV - Ponto de vista do entrevistador. A pontuação do QSAP é feita em três níveis: (a) a cotação dos itens do questionário em termos de intensidade; (b) a cotação do conjunto de cada parte e a equivalência dos pontos obtidos em relação ao total de pontos possíveis - índices TOT (PA) e TOT (FO); (c) os índices gerais do questionário - índices ITOT (PA), ITOT (FO) e IAE. Os dois primeiros referem-se ao total de pontos associados respectivamente à potência aeróbia e à força (ITOT - índices totais), enquanto o IAE (ou índice de autonomia exprimida) representa o resultado geral do questionário.
No TSMP, as idosas realizavam marcha de 800 metros transportando pesos específicos para o sexo feminino $(6,5 \mathrm{~kg})$, de acordo com as normas e recomendações originais, compreendendo três fases: a) fase de pré-fadiga - imóvel e de pé, o indivíduo sustenta pesos predeterminados para seu sexo em cada uma das mãos (8kg para homens e 6,5kg para mulheres) durante três minutos, ao fim dos quais é autorizado a começar a caminhada. Os pesos devem ser bem calibrados, de maneira a proporcionar boa distribuição bilateral das cargas. O ponto de contato entre os pesos e as mãos deve ser revestido de material macio (tecido, fitas acolchoadas etc), para que o teste não seja interrompido em função de dor nas mãos. b) fase de trabalho - o indivíduo é convidado a percorrer, sem correr, 800m 'o mais rapidamente possível sem colocar em risco a saúde', portando os pesos específicos ao seu sexo.

O TSMP produz o índice de autonomia potencial (IAP), cuja determinação é feita a partir de quatro variáveis: o índice de massa corporal (IMC) $\left(\mathrm{kg} / \mathrm{m}^{2}\right)$; o tempo de percurso na fase de trabalho (T-800) (s); uma categoria ordinal correspondente ao número de pausas realizadas (S-PAUSA); a porcentagem da FC máxima atingida no teste (\%FCMáx). As categorias adimensionais para as pausas são: nenhuma pausa (SPAUSA $=0$ ), uma a duas pausas $(S-P A U S A=1)$, três pausas (S-PAUSA = 2), quatro pausas ( $($-PAUSA $=3$ ), mais que quatro pausas (S-PAUSA = 4), não transportar os pesos desde o início do teste (S-PAUSA $=5)$. Se o indivíduo não é capaz de percorrer os $800 \mathrm{~m}$, mesmo com as mãos livres, excepcionalmente o tempo aos $400 \mathrm{~m}$ pode ser utilizado para estimar o T-800 (T-800 = T-400 × 2). Esse procedimento é possível em função da alta correlação entre T-400 e T-800 ( $r>0,90 ; p<0,001)^{(19)}$. Finalmente, a \% FC é calculada pela fórmula ( $\left.F C_{\text {teste }} \times 100\right) / F C_{\text {max }} \cdot A F C_{\text {teste }}$ corresponde à maior $\mathrm{FC}$ observada durante o teste. $\mathrm{AFC} \mathrm{C}_{\max }$ Corresponde à $F C$ máxima prevista de acordo com a idade (220 - idade).

O quociente entre IAP e IAE permite o cálculo do índice sênior de autonomia de ação (ISAC), considerado o resultado final do SysSen. São considerados como autônomos os sujeitos cujo ISAC for maior ou igual a 1. Na verdade, o ISAC é calculado pela razão entre valores corrigidos do IAP e IAE. Essa correção deve-se à adaptação dos valores obtidos pelos índices a uma unidade comum, a saber, a idade cronológica expressa em anos. O fato de existir apenas um índice para o QSAP e dois para o TSMP (feminino e masculino) impôs a adoção dessa estratégia para que os dados pudessem ser estatisticamente compatíveis, permitindo o estabelecimento de uma razão entre os dois índices. Assim, antes do cálculo do ISAC, cumpre-se a etapa de ajustamento dos índices parciais. As fórmulas para o cálculo do IAE, IAP e ISAC são apresentadas no quadro 1.

Quadro 1. Fórmulas para cálculo dos índices parciais e total do SysSen.

\begin{tabular}{|c|}
\hline Índice sênior de autonomia exprimida (IAE) \\
\hline $\begin{array}{c}6,99 \times \mathrm{IAE}_{\text {bruto }}+69,88 \\
\mathrm{IAE}_{\text {bruto }}=7,496 \times \text { ITOT }(\mathrm{PA})+7,899 \times \text { ITOT }(\mathrm{FO})-3,423 \\
\text { ITOT }(\mathrm{PA})=\text { somatório dos índices parciais das partes do QSAP para PA } \\
\text { ITOT }(\mathrm{FO})=\text { somatório dos índices parciais das partes do QSAP para FO }\end{array}$ \\
\hline Índice sênior de autonomia potencial (IAP) \\
\hline 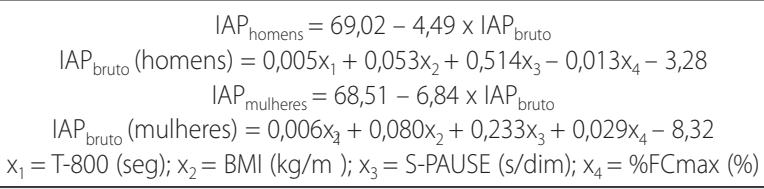 \\
\hline Índice sênior de autonomia de ação (ISAC) \\
\hline $\begin{array}{c}I S A C=I A P_{\text {corr }} / I A E_{\text {corr }} \\
I A P_{\text {corr }} \text { (mulheres) }=1,48 \times I A P_{\text {mulheres }}-52,43 \\
I A E_{\text {corr }} \text { (mulheres) }=2,04 \times \mid A E-91,65 \\
\mid A P_{\text {corr }} \text { (homens) }=2,23 \times \mid A P_{\text {homens }}-102,86 \\
\mid A E_{\text {corr }} \text { (homens) }=1,37 \times \mid A E-46,10\end{array}$ \\
\hline
\end{tabular}


Todas as avaliações foram feitas no Laboratório de Fisiologia do Exercício (Lafex) da Universidade Federal do Espírito Santo (Ufes) e no módulo do Serviço de Orientação ao Exercício (SOE) da Praça dos Desejos, localizado na Cidade de Vitória. Antes do TSMP era aferida a pressão arterial das idosas pelo método auscultatório, respeitando as orientações das V Diretrizes Brasileiras de Hipertensão Arterial(21), sendo utilizado esfigmomanômetro de coluna de mercúrio (Glicomed Premium, Rio de Janeiro, RJ, Brasil) no Lafex e Missouri (Cirúrgica Gervásio, Belo Horizonte, MG, Brasil) no SOE. Em seguida, era aferida a frequência cardíaca de repouso ( $\mathrm{FCr}$ ) por meio da palpação digital no punho (artéria radial), em um período de 15 segundos e multiplicado o valor encontrado nesse período por quatro para definir o valor da frequência em um minuto. No Lafex o peso e a estatura foram medidos com balança digital da marca Soehnle ${ }^{\circledast}$ e estadiômetro da marca Asimed ${ }^{\circledR}$ (Goiânia, GO,Brasil), respectivamente. Essas mesmas variáveis foram aferidas no SOE com a balança e estadiômetro Welmy (Santa Bárbara D'Oeste, SP, Brasil). Os locais de realização do TSMP foram quadras esportivas na Ufes e na Praça dos Desejos. Em ambas foi delimitado um percurso de 50 metros, percorrido por 16 vezes para que se alcançassem os 800 metros.

\section{TRATAMENTO ESTATÍSTICO}

A Anova de duas entradas para medidas repetidas seguida de verificação post-hoc de Fisher foi utilizada para a comparação entre IAE, IAP e idade cronológica dos grupos GE e GNE. Já para comparar os resultados concernentes às necessidades para autonomia relacionada às diferentes qualidades físicas focalizadas pelo QSAP (FO e PA), dentro de cada grupo (GNE e GE), utilizamos o teste $t$ de Student para amostras pareadas. Por fim, possíveis diferenças entre GNE e GE para os resultados nas diferentes partes do QSAP (TOTI, TOT II, TOT III e TOT IV para potência aeróbia e força de membros superiores) foram comparados pelo teste de KruskallWallis, seguido do teste de Wilcoxon a título de verificação post-hoc. Para a comparação intragrupo referente às partes do QSAP utilizou-se o teste de Friedman complementado pelo teste de Wilcoxon, quando necessário. O teste $t$ de Student para amostras independentes foi utilizado para comparar as médias das variáveis IMC, tempo total do percurso, número de pausas, FC de repouso e FC após a caminhada (imediatamente) entre GNE e GE. O nível de significância adotado em todos os testes foi de $p \leq$ 0,05 . Os cálculos foram realizados com auxílio do programa GB-Stat. v. 6.5 para Windows (Dynamic Microsystems, Silver Spring, MT, EUA).

\section{RESULTADOS}

As características amostrais para GE e GNE são apresentadas na tabela 1. Não houve diferença significativa entre as variáveis.

A figura 1 apresenta os resultados da Anova para a comparação en-

Tabela 1. Comparação das características antropométricas das idosas praticantes (GE) e não praticantes (GNE) de exercício físico regular. Valores apresentados como média \pm desvio padrão.

\begin{tabular}{c|c|c|c|c}
\hline & Idade (anos) & Estatura (m) & Peso (kg) & IMC (kg/m²) \\
\hline GNE & $68 \pm 5$ & $1,54 \pm 0,05$ & $65,7 \pm 14,6$ & $27,5 \pm 5,4$ \\
\hline$G E$ & $69 \pm 6$ & $1,56 \pm 0,04$ & $67,4 \pm 10,1$ & $27,7 \pm 3,6$ \\
\hline$P$ & 0,56 & 0,28 & 0,65 & 0,86 \\
\hline
\end{tabular}

tre IAE, IAP e idade cronológica no GE e GNE. Observa-se condição física superior em $G E$, representada pelo maior IAP $(P=0,01)$. Por outro lado, as necessidades em termos de atividades físicas para força e resistência foram próximas para os dois grupos analisados ( $P=0,85)$. Não houve diferenças significativas entre os grupos na comparação dos valores médios do ISAC (GE: ISAC =0,88 vs. GNE: ISAC =0,86; P = 0,62).

A figura 2 exibe a comparação entre os resultados de ITOT (PA) e ITOT (FO) em GE e GNE. Não houve diferença quando se comparou ITOT (PA) com ITOT (FO), tanto em GNE (P=0,11), quanto em GE (P =

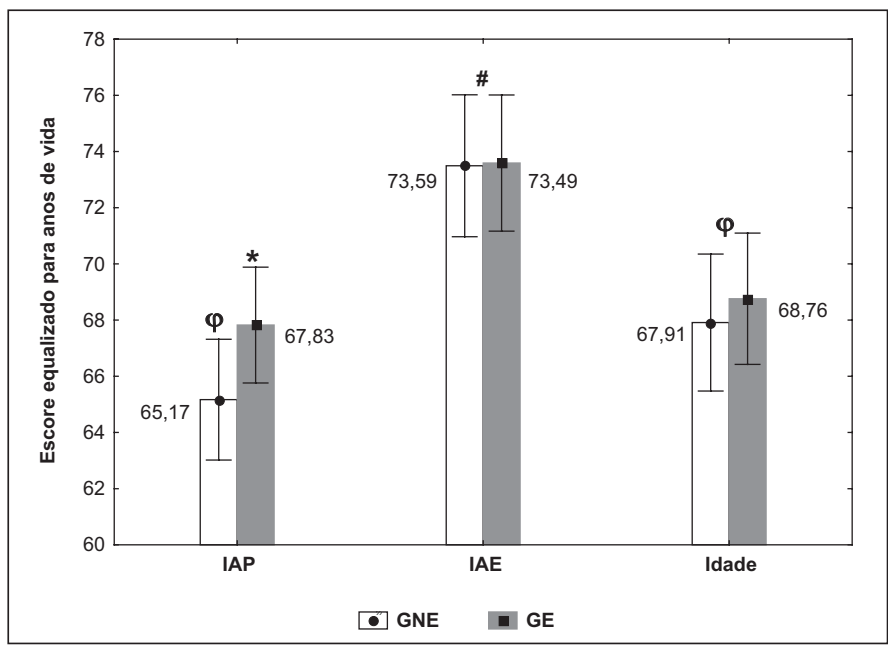

Figura 1. Valores médios e resultados da Anova de duas entradas para medidas repetidas seguida de verificação post-hoc de Fisher para IAE, IAP e idade cronológica de grupos praticantes (GE) e não praticantes (GNE) de exercício físico regular. IAP: índice de autonomia potencial; IAE: índice de autonomia exprimida. * Diferença significativa entre GNE e GE para o IAP $(P<0,05)$; \# Diferença significativa entre o IAE dos grupos GNE e GE em relação a todos os demais grupos (IAP e Idade) $(P<0,05)$; $\varphi$ Diferença significativa entre IAP (GNE) e Idade (grupos GE e GNE) $(P<0,05)$. As barras de dispersão representam intervalos de confiança a 95\%.

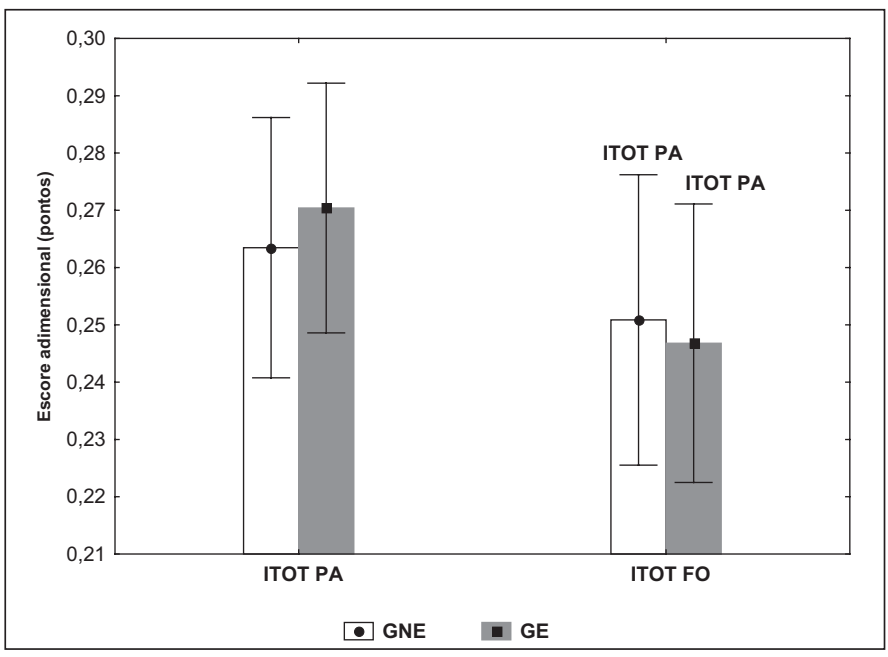

Figura 2. Valores médios e resultados da Anova de duas entradas para medidas repetidas seguida de verificação post-hoc de Fisher para ITOT (PA) e ITOT (FO) nos grupos praticantes (GE) e não praticantes (GNE) de exercício físico regular. Não houve diferença significativa entre os grupos para um mesmo índice $(p>0,05)$. Os índices sobrescritos indicam diferença para este índice em um mesmo grupo $(p<0,05)$. As barras de dispersão representam intervalos de confiança a 95\%.

0,42), apesar de haver vantagem absoluta em favor da PA em ambos os casos. Também não se constataram diferenças significativas entre GNE e GE para ITOT (PA) $(P=0,49)$ e ITOT $(F O)(P=0,85)$.

Na figura 3 apresentam-se os resultados referentes às diferentes partes do QSAP. A comparação intergrupos (GE vs. GNE) revelou diferença significativa para os TOT I (PA/FO) em relação aos TOT II (PA/ FO) e TOT IV (PA/ FO). A comparação intragrupos (dimensões do QSAP para GE ou GNE) revelou, em termos de PA e FO, diferenças significativas entre algumas partes do QSAP. OTOT I (PA/FO) do GNE teve diferenças significativas em relação ao TOT II, havendo vantagem para TOT I. No GE a mesma situação foi observada, incluindo diferença significativa entre TOT I e TOT III.

Os resultados do TSMP são apresentados na tabela 2. As médias para as variáveis N-PAUSA, FCteste, \%FCmáx, PAS e PAD foram semeIhantes entre GE e GNE. Já o tempo percorrido mostrou-se significativamente menor em GE do que em GNE (GNE =679s vs. 631s; $P=0,05$ ). Houve também diferença significativa para FCr entre os dois grupos $(\mathrm{GNE}=83 \mathrm{bpm}$ vs. 73bpm; $\mathrm{P}=0,001)$. 


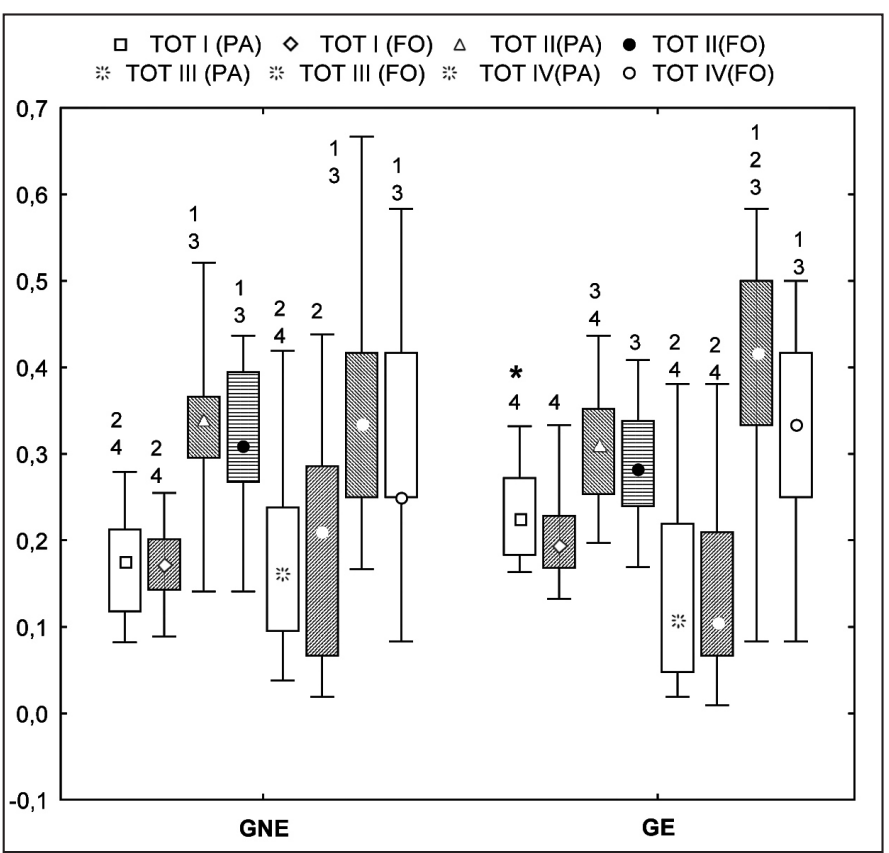

Figura 3. Estatística descritiva e resultados da Anova Friedman seguida de verificação post-hoc de Wilcoxon para os índices parciais do QSAP nos grupos praticantes (GE) e não praticantes (GNE) de exercício físico regular. * Diferença significativa entre GNE e GE para uma mesma parte do questionário $(P<0,05)$. Os algarismos indicam diferença significativa daquela parte do questionário em relação às partes por eles representadas em um mesmo grupo $(p<0,05)$. Por exemplo, o índice TOT I (PA) no GNE foi significativamente menor que os índices TOT (PA) da Parte II e Parte IV do QSAP. Os valores intermediários representam as medianas, as caixas representam os percentis 25 e 75 (quartis) e as barras indicam os valores mínimo e máximo observados.

Tabela 2. Resultados dos dados do teste sênior de caminhar e transportar (TSMP), realizado por idosas praticantes e não praticantes de exercícios físicos regulares. Os valores são apresentados como média \pm desvio padrão. GNE $(n=21)$ e $G E(n=27)$.

\begin{tabular}{c|c|c|c|c|c|c|c}
\hline & Tempo & No pausas & FC (bpm) & $\%$ FCmáx & $\begin{array}{c}\text { FCr } \\
(\mathbf{b p m})\end{array}$ & $\begin{array}{c}\text { PAS } \\
(\mathbf{m m H g})\end{array}$ & $\begin{array}{c}\text { PAD } \\
(\mathbf{m m H g})\end{array}$ \\
\hline GNE & $679 \pm 78,0$ & $0,62 \pm 1,2$ & $119 \pm 17$ & $77 \pm 9,9$ & $83 \pm 9$ & $121 \pm 16$ & $78 \pm 13$ \\
\hline$G E$ & $631 \pm 91,1$ & $0,52 \pm 1,2$ & $113 \pm 22$ & $74 \pm 12,6$ & $73 \pm 11$ & $126 \pm 14$ & $80 \pm 8$ \\
\hline$P$ & 0,05 & 0,77 & 0,27 & 0,44 & 0,001 & 0,20 & 0,52 \\
\hline
\end{tabular}

A figura 4 apresenta a plotagem individual de todos os casos para o ISAC (Isacograma), permitindo visão geral do resultado final do teste em ambos os grupos, dado o ponto de corte de 1,0, mínimo necessário para autonomia de ação adequada. Apesar de não existir diferença significativa entre as médias do ISAC entre GE e GNE, observa-se que a quantidade de idosas que alcançaram esse limiar em GE foi praticamente o dobro daquelas em GNE.

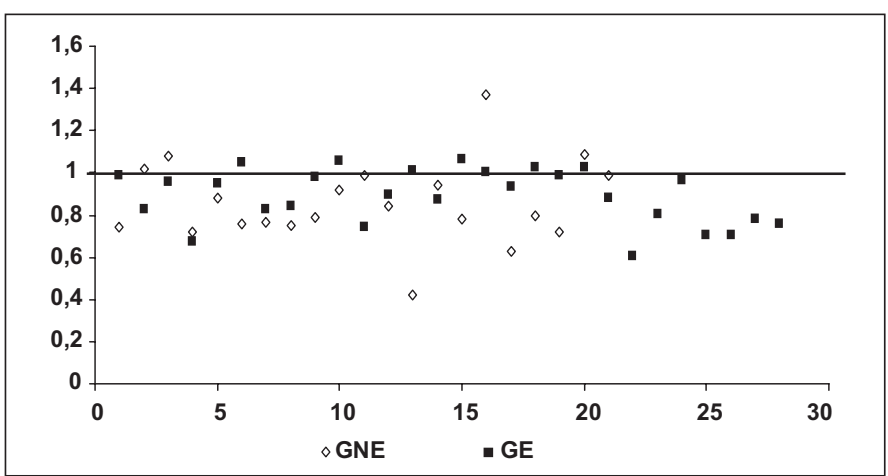

Figura 4. Isacograma - Representação gráfica dos resultados do ISAC para cada indivíduo nos grupos praticantes de exercício físico regular (GE) e não praticantes de exercício físico regular (GNE), bem como a relação com o ponto de corte para a autonomia de ação (ISAC = 1,0), representado pela linha contínua.

\section{DISCUSSÃO}

O presente estudo teve por objetivo comparar os resultados do SysSen em mulheres idosas praticantes e não praticantes de exercícios físicos de forma regular. Não houve manipulação ou controle estrito da variável "exercício" (por exemplo, sua intensidade, frequência, duração etc). Apesar dessa limitação, os principais resultados indicaram que um maior IAP por parte de GE não se traduziu em autonomia de ação significativamente superior, ao menos quando traduzida pelo ISAC. Por outro lado, a quantidade de idosas que alcançaram um ISAC acima do mínimo considerado necessário para serem classificadas como autônomas foi nitidamente maior para o grupo que se exercitava. Os resultados do QSAP revelaram peso maior das atividades realizadas efetivamente pelas idosas do GE em comparação com GNE. Contudo, não foi encontrada diferença para as necessidades mais gerais em termos de força de membros superiores e potência aeróbia para a execução das atividades ligadas à percepção de vida autônoma, uma vez que o IAE foi similar entre os grupos.

Os grupos eram similares em relação à idade, peso e estatura (tabela 1). Não é possível, porém, afirmar que as idosas que compuseram o GNE eram absolutamente sedentárias - exigiu-se apenas que estivessem sem praticar regularmente exercício físico durante seis meses. No entanto, há evidências de que três meses seriam suficientes para pessoas idosas sofrerem redução da aptidão física com o destreinamento(22).

Uma vez corrigido para a idade, o índice relacionado à aptidão física (IAP) revelou-se menor que o esperado entre as mulheres que não praticavam exercício físico regularmente. Por outro lado, as necessidades em termos de atividades físicas refletidas pelo IAE não foram diferentes entre os grupos e, ao serem corrigidas para unidades de idade cronológica, foram sempre maiores que a idade real. Resultados similares foram encontrados por Farinatti et al. ${ }^{(16)}$. A diferença entre os dois estudos é que, apesar de os presentes resultados terem identificado menor tempo final para a execução do TSMP e maior IAP para GE em comparação com GNE (tabela 2), não foi possível observar diferença significativa entre o ISAC dos dois grupos.

A melhoria do tempo para percorrer $800 \mathrm{~m}$ decorre, provavelmente, de melhor capacidade cardiorrespiratória e força muscular ${ }^{(1,23)}$. Desse modo, seria razoável pensar que as idosas do GE teriam se beneficiado dos efeitos da prática regular de exercícios físicos ${ }^{(9,10,24)}$. Além disso, as idosas fisicamente ativas percorreram a distância com menor FC absoluta (GNE $=119 \pm 17 \mathrm{bpm} v \mathrm{~s}$. GE $=113 \pm 22 \mathrm{bpm}$ ) e menor percentual representativo da FCmáx (GNE $=77 \pm 9,9 \mathrm{bpm}$ vs. GE $=74 \pm 12,6 \mathrm{bpm})$, apesar de diferenças significativas não terem sido constatadas para essas variáveis.

O melhor desempenho no TSMP pelo grupo fisicamente ativo reforça a consistência desse componente do SysSen. A eficiência da caminhada teria relação forte com a percepção de saúde ${ }^{(25)}$, assim como é considerada como bom preditor de dependência futura ${ }^{(26)} \mathrm{e}$ investe-se de boa associação com a função muscular de forma geral(27). Um desempenho superior nas variáveis do TSMP (velocidade de caminhada, número de pausas, menor FC durante o teste), independentemente do próprio IAP, deve ser visto como informação clinicamente relevante para a manutenção da autonomia funcional de idosos, principalmente em idades mais avançadas.

A comparação entre GE e GNE para o IAE revela que o fato de as idosas praticarem exercício não modifica o que elas expressam no tocante às necessidades para uma vida autônoma. É possível que este fato se deva ao meio ambiente em que elas vivem, apresentando exigências semelhantes em termos de PA e FO, o que, aliás, é ratificado pelos resultados da Parte II do QSAP (figura 3). Nos dois grupos a pontuação para as necessidades, em termos de atividades físicas compatíveis com uma vida autônoma, foi razoavelmente equilibrada entre as partes do questionário, a não ser para a Parte II, cuja cotação foi significativamente maior que aquelas das Partes I e III. Isso sugere que o meio ambiente físico, além de impor demandas parecidas em termos de força e potência aeróbia para uma vida autônoma, constitui principal fonte de obstáculos para a independência funcional das idosas observadas. 
Nesse contexto, o fato de GE ter obtido valores mais elevados para o IAP é relevante em termos práticos, já que, em princípio, esse grupo teria melhor condição de fazer face às exigências ambientais. Jóia et al. ${ }^{(5)}$ relataram que o fator mais importante relacionado à satisfação com a própria vida entre idosos de Botucatu/SP, era a capacidade de se desincumbir bem das atividades de vida diária, indicando aproximação direta com que eles consideravam ser a saúde. Porém, não foi encontrada nenhuma relação com a prática de atividades físicas. Rigo e Teixeira ${ }^{(28)}$ reforçam esses resultados, indicando que o bem-estar na velhice estaria relacionado aos julgamentos que os próprios idosos fazem sobre as suas possibilidades de ação.

A comparação dos índices parciais do QSAP referentes às demandas especificamente associadas à força de membros superiores - ITOT (FO) - e potência aeróbia - ITOT (PA) - revelou demandas similares, já que não se identificaram diferenças estatísticas entre os seus resultados. Os valores medianos, contudo, foram ligeiramente superiores para ITOT (PA). Essa tendência aproxima-se dos resultados de estudo prévio(16) , em que pequena diferença favorável às necessidades impostas pela potência aeróbia foi identificada em grupos de mulheres idosas fisicamente ativas, brasileiras e belgas. A possibilidade de esse perfil constituir um padrão entre idosas, independentemente do nível de atividades físicas praticadas, ainda está por ser confirmada.

É importante notar que não houve discrepância entre a Parte ॥ (exigências do ambiente) e a Parte IV (ponto de vista do entrevistador) do QSAP em GNE, mas sim em GE. Isso indica que no grupo que não se exercitava obteve-se equilíbrio entre a avaliação das entrevistadas sobre as demandas físicas associadas às características ambientais das idosas e o julgamento do próprio entrevistador. Já no grupo fisicamente ativo, aparentemente as entrevistadas subestimaram as demandas ambientais. Isso pode acontecer e não compromete a consistência do questionário, uma vez que o descompasso não se refletiu no IAE. O mesmo se deu, por exemplo, no estudo de Farinatti et al. ${ }^{(16)}$, no qual idosas brasileiras subestimaram sua realidade, enquanto as belgas a superestimaram. A Parte IV do QSAP foi pensada justamente para compensar possíveis problemas de avaliação em relação à própria realidade, notadamente no que diz respeito ao ambiente físico. $\mathrm{O}$ fato de idosas ativas considerarem as dificuldades ambientais menos importantes apenas reflete o quanto sua autoconfiança pode ter sido exacerbada por condição física melhor. Assim, o indivíduo que não pratica exercícios regulares pode perceber um dado esforço de forma diferente que pessoas mais acostumadas a esforços de maior intensidade.

Os resultados referentes ao índice mais geral do SysSen, o ISAC, são o reflexo das considerações até aqui tecidas. Apesar de não ter sido

\section{REFERÊNCIAS}

1. Kirkendall DT, Garrett Jr E. The effects of aging and training on skeletal muscle. Am J Sports Med 1998;26:598-602. 2. Farinatti PTV, Ferreira MS. Saúde, promoçăo da saúde e educaçăo física: conceitos, princípios e aplicaçōes. Rio de Janeiro: EdUERJ, 2006.

3. Farinatti PTV. Teorias biológicas do envelhecimento: do genético ao estocástico. Rev Bras Med Esporte 2002:8:129-38.

4. Suzanne G, Leveille JM, Guralnik LF, Jean AL. Aging successfully until death in old age: opportunities for Increasing active life expectancy. Am J Epidemiol 1999;149:654-64.

5. Jóia LC, RuizT, Donalísio MR. Grau de satisfação com a saúde entre idosos do município de Botucatu, estado de São Paulo, Brasil. Epidemiol Serv Saúde 2008;17:187-94.

6. Papaléo N, Ribeiro J. Envelhecimento: Desafio na transiçăo do século. In: Papaléo N. Gerontologia. São Paulo: Atheneu, 1996;3-12.

7. Buriti M, Campelo CP. In: Witter G. Envelhecimento: Referências teóricas e pesquisa. São Paulo: Alínea, 2006;119-29. 8. Simăo R. Treinamento de força na saúde e qualidade de vida. Săo Paulo: Phorte, 2004

9. Silva N, Farinatti PTV. Influência de variáveis do treinamento contra-resistência sobre a força muscular de idosos: uma revisăo sistemática com ênfase nas relações dose-resposta. Rev Bras Med Esporte 2007;13:60-6.

10. Schettino L, Pereira R, Machado M, Monteiro A, Dantas PM. Estudo comparativo da força e autonomia em idosas-sedentárias versus ativas. Ter Man 2008;6:32-6.

11. Janssen I, Baumgartner RN, Ross R, Rosenberg $\mathbb{H}$, Roubenoff R. Skeletal muscle cutpoints associated with elevated physical disability risk in older men and women. Am J Epidemiol 2004;159:413-21.

12. Matsudo SM, Matsudo VKR, Barros Neto TL. Impacto do envelhecimento nas variáveis antropométricas, neuromotoras e metabólicas da aptidão física. Rev Bras Ciên e Mov 2008;4:21-32.

13. Amasa BF, Marie RH, Kurt CS, Atwood DG, Linda SN, Paul KJ. Sustained personal autonomy: a measure of successful aging. J Aging Health 2000;12:470-89.

14. Farinatti PTV. Avaliaçăo da autonomia do idoso: definição de critérios para uma abordagem positiva de um modelo de interaçăo saúde/autonomia. Arquivos de Geriatria e Gerontologia 1997;1:31-8. identificada diferença significativa entre a autonomia de ação das idosas de GE e GNE, houve ligeira vantagem absoluta em favor das primeiras (GE: 0,86 vs. GNE: 0,89; $p=0,55$ ). O fato de, em termos estatísticos, as diferenças entre os valores médios do ISAC em grupos com características diferentes não serem significativas, não é surpresa. Pela lógica do SysSen, é perfeitamente possível que sujeitos com aptidão física inferior sejam considerados mais autônomos (maior ISAC) do que outros com melhor condição atlética. Basta que essa maior aptidão (traduzida pelo IAP) fique aquém das necessidades levantadas pelo QSAP (refletida pelo IAE). No presente caso, a vantagem identificada para o IAP não foi suficiente para alterar de forma mais impactante o ISAC, provavelmente porque os valores do IAE compensaram a diferença. Mais importante do que a comparação entre médias do ISAC em GE e GNE, talvez seja o quadro ilustrado pelo Isacograma exibido na figura 4: em GNE, das 21 mulheres observadas, apenas seis (29\%) situaram-se acima da linha demarcatória do ISAC $\geq 1,0$. Dentre as 27 idosas do GE, por outro lado, 10 (37\%) ultrapassaram esse ponto de corte. Considerando o conjunto dos dados, é plausível que esses resultados tenham decorrido de um déficit de aptidão físico-funcional mais frequente em GNE que em GE, já que os resultados obtidos para o IAE foram similares entre os grupos.

\section{CONCLUSÃO}

Idosas praticantes e não praticantes regulares de exercícios físicos exibiram níveis similares de autonomia de ação traduzidas pelo ISAC. Contudo, a quantidade de idosas que foram consideradas autônomas na lógica do SysSen (ISAC $\geq 1,0$ ) foi maior em $G E$, que apresentou superioridade significativa em relação à aptidão física, de acordo com os resultados do TSMP (IAP superior).

Os resultados do QSAP (traduzidos pelo IAE) para as necessidades de atividades físicas realizadas, impostas pelo meio ambiente e referidas como importantes para a percepção de uma vida autônoma, foram similares nos dois grupos.

No que diz respeito especificamente ao QSAP, houve equilíbrio entre as necessidades associadas à força de membros superiores e potência aeróbia (índices ITOT (FO) e ITOT (PA)). Por outro lado, em ambos os grupos a pontuação aferida para a Parte II do questionário foi significativamente superior ao observado na Parte I e na Parte III. O impacto ambiental, portanto, revelou-se mais importante na determinação das necessidades relacionadas à autonomia de ação dos grupos observados, do que possíveis frustrações derivadas da não realização de atividades por eles valorizadas.

Todos os autores declararam não haver qualquer potencial conflito de interesses referente a este artigo.

15. Farinatti PTV. Proposta de um Instrumento para avaliaçăo da autonomia do Idoso: o sistema senior de avaliaçăo da autonomia de ação (SysSen). Rev Bras Med Esporte 2000;6:224-40.

16. Farinatti PTV, Assis BF, Silva NS. Estudo comparativo da autonomia de açăo de idosas participantes de programas de atividade física no Brasil e Bélgica. Rev Bras Cineantropom Desempenho Hum 2008:10:107-14.

17. Mattos M, Farinatti PTV. Influência do treinamento aeróbio com intensidade e volume reduzidos na autonomia e aptidão físico-funcional de mulheres idosas. Rev Port Cien Desp 2007;7:100-8.

18. Laville C, Dionne A. A construçăo do saber: manual de metodologia da pesquisa em ciências humanas. Porto Alegre: Artes Médicas Sul, 1999.

19. Farinatti PTV, Vanfraechem JHP. Descriçăo e desenvolvimento do aeste de'caminhar e transportar'. Artus 1999;19:81-99.

20. Farinatti PTV, Vanfraechem JHP, Clemen D. Descriçāo e construção do questionário senior de atividades físicas para idosos (QSAP). Rev Bras Med Esp 1998:4:45-54.

21. Sociedade Brasileira de Cardiologia.V Diretrizes brasileiras de hipertensão arterial. Arq Bras Cardiol 2007:89:e-24-e-79.

22. Carvalho MJ, Marques E, Mota J. Training and detraining effects on functional fitness after a multicomponent training in older women. Gerontology 2008;Published Online:June 19.

23. Marcelino VR. A estruturaçăo de um programa de trabalho resistido para o idoso: uma proposta de intervençăo. 2003; Dissertaçăo (mestrado) - Universidade Estadual de Campinas, Faculdade de Educaçăo Física. Campinas.

24. Krause MP, Buzzachera CF, Hallage T, Pulner SB, Silva SG. Influência do nível de atividade física sobre a aptidăo cardiorrespiratória em mulheres idosas. Rev Bras Med Esporte 2007;13:97-102.

25. Ferreira FFP, IZzo H, Filho WJ. Impacto da capacidade física da saúde percebida entre idosos em velhice avançada. Saúde Coletiva 2007:4:154-7.

26. Woo J, Ho SC, Yu AL. Walking speed and stride length predicts 36 months dependency, mortality, and institutionalization in Chinese aged 70 and older. J Am Geriatr Soc 1999;47:1257-60.

27. Nobre LC, Farinatti PTV. Amplitude e cadência do passo e componentes da aptidăo muscular em idosos: um estudo correlacional multivariado. Rev Bras Med Esporte 2004;10:389-94.

28. Rigo MLNR, Teixeira DC. Efeitos da atividade física na percepção de bem-estar de idosas que residem sozinhas e acompanhadas. UNOPAR Cient Ciênc Biol Saúde 2005,7:13-20. 